\title{
Association between Theory of Mind and Peer Problems
}

\author{
Maham Abdullah $^{1}$, Sadaf Rehman ${ }^{2}$, Sumbal Nawaz ${ }^{3}$, Shamaila Asad ${ }^{4}$, Samia Khalid ${ }^{1}$
}

\section{Abstract}

The current study was designed to investigate the relationship between theory of mind (ToM) development and peer problems in Pakistani children $(N=80)$. The non-probability purposive sampling technique with survey research design had been used for data collection. Pakistani children with age ranged 4-6 years $\left(M_{\text {age }}=5.29\right)$ were recruited who took two false belief tasks. To tap into peer relationship of these children, their parents completed strength and difficulty questionnaire. For demographic variables, descriptive statistics was used. Pearson product correlation and linear regression were used to test the hypothesis. Results revealed that performance of 6 years 6 months and older was above chance on all false belief tasks, supporting the universality of ToM development with different age ranges in different cultures. Also, theory of mind negatively predicted peer relationship problems of this sample, revealing real life implication of mentalizing for interaction in social world. Research indicated that false belief comprehension is key to better social adjustment and the participants of this study also showed that a child's understanding of mental state terms is critical for better social adaptation.

Keywords: Theory of Mind, Peer Problems, False Belief, Development, Children

Received: 07 November 2021; Revised Received: 05 December 2021; Accepted: 08 December 2021

${ }^{1}$ Lecturer, Riphah Institute of Clinical \& Professional Psychology, Riphah International University, Lahore Campus, Pakistan.

${ }^{2}$ Research Associate, Riphah Institute of Clinical \& Professional Psychology, Riphah International University, Lahore Campus, Pakistan.

${ }^{3}$ Assistant Professor, School of Social Sciences \& Humanities, National University of Sciences \& Technology, Islamabad, Pakistan.

${ }^{4}$ Assistant Professor, Riphah Institute of Clinical \& Professional Psychology, Riphah International University, Lahore Campus, Pakistan.

\section{Corresponding Author Email:} maham.abdullah@riphah.edu.pk

\section{Introduction}

The purpose of this study was to investigate the age when theory of mind develops in Pakistani children and the relationship between age and theory of mind in 4 to 6 years old children. The preschoolers undergo significant changes in their social cognitive development, as they start showing knowledge about memory and attention, develop logical reasoning skills, communication skills, language and the ability to understand mental states like beliefs, desire and knowledge. They can also differentiate between their own and others mental states, which is known as theory of mind (ToM) and with this ability, they can understand, explain and predict one's own behaviors and that of others which are evident and result in everyday actions of human beings (Malle, 2018). Imagine a person who stands up from living room and walks towards his pantry to get a chocolate cookie, because he may be hungry, he likes chocolate cookie and thinks he can find one

This article is distributed under the terms of the Creative Commons Attribution Non Commercial 4.0 License (http://www.creativecommons.org/licenses/by-nc/4.0/) which permits nonCommercial use, reproduction and distribution of the work without further permission provided the original work is attributed as specified. 
in his pantry, and if he is unable to find it, one can predict that it can upset him. As evident, one can propose different explanations of single action or behavior. Hence, theory of mind helps us in making predictions and explanations of other's actions (Wellman, 2007). During preschool years of development, children develop false belief understanding and come to terms with the understanding that what a person thinks is most important in controlling their actions (Wellman, 2012), other than just reality. And to test theory of mind, false belief is referred as a litmus test to tap ToM as theory of mind cannot be tested directly.

Theory of mind is a key milestone in the development of children and the understanding of theory of mind is important to take place in the initial years, as it leads to further complex and critical thinking in later years (Devine et al., 2016). Few researchers have claimed that theory of mind develops around the age of 5 years in Western as well as non-Western cultures, marking the universality of ToM development (Callaghan et al., 2005). As per Liu et al. (2008), in comparison to children of West, there is a lag of 2 years in ToM development of Chinese and Hongkong children and similar results are there for Japanese and Korean children (Oh \& Lewis, 2008). Similar is the case for Pakistani children. According to research done by Nawaz et al. (2015), at the age of 5 years performance on theory of mind tasks is at chance, and these findings were replicated (Nawaz \& Lewis, 2017). To fill this gap, one objective of the study is to explore at what age children in Pakistan achieve theory of mind development. This finding will contribute to the understanding if ToM development is universal in timing or not.

The normative development of theory of mind is critical, as it is important in the evolution of social relations, the competency of a child to interact in richer and complex social interactions (Patnaik, 2008). Theory of mind is related to peer popularity and happier children in schools, because they are competent socially, as rated by their teachers (Astington et al., 2010). ToM has implications in the development of academic skills of children, as it impacts the learning process of children (Patnaik, 2008). The ability of theory of mind helps children to resolve their conflicts with peers, and the reason might be that ToM understanding may help them to communicate better, let's them explain their own behavior and they can understand why others did what they did (Astington, 2008). This ability develops into better social relations in adult life.

Theoretically, the major role of theory of mind is the benefits in social relations for all children. In particular, peer interactions are the most important outcome of developed theory of mind ability (Peterson et al., 2016). As the ability of ToM understanding enables us to explain the intentions, desires and beliefs behind the behaviors of others, which basically allows the children to improve social cognitive development.

Furthermore, due to the importance of peer relations in the development of children, many researchers over the past few years have been interested in finding the predictors of peer relations. Recently, Slaughter et al. (2015) conducted a meta-analysis, according to which, there is a significant, positive association between children's theory of mind ability and their relationship with peers. These studies support the notion that children who are competent in understanding the mental states of others, are effective in social behaviors, which relates to them being well liked by their peers. Whereas, children whose understanding of others' mental states is underdeveloped or relatively poor, are seen as less capable socially. This analysis is the first and only analysis confirming that the performance on theory of mind tasks is related to the everyday social lives of the children, supporting the real-life significance 
of theory of mind (Slaughter et al., 2015). In this study, the relationship between theory of mind and peer problems will be investigated in Pakistani children. Aggression is also linked with peer relationship (Chen et al., 2012) and to know if theory of mind and peer relations are linked, it is important to keep aggression as a control variable, to investigate if theory of mind can predict peer problems independent of aggression.

\section{Rationale \& Objectives}

Theory of mind is an important concept and is crucial for intelligible social interactions. According to cross cultural research, there in cultural variation in the development of theory of mind, also there is a developmental lag in non-Western cultures like Iran and China on the understanding of false belief (Wellman \& Liu, 2004), including Pakistan (Nawaz et al., 2014). The age when Pakistani children develop theory of mind is not known till date, which is a major gap in literature. Knowing the significance of the theory of mind, and the presence of developmental lag, makes it important to address this gap hence, this study will be a one-step contribution in exploring the age at when theory of mind develops in Pakistani children. As 5-6 years old children are about to enter primary schools, there is high demand for children to begin and maintain positive interpersonal relations with peers and teachers (Campbell \& Stauffenberg, 2008).

Moreover, the peer problems have never been studied in the context of theory of mind in Pakistan; if there is any relation between theory of mind and peer problems. And it is pertinent to study peer problems with reference to theory of mind to understand the cognitive components related to social understanding. The understanding that how theory of mind abilities might impact social development in Pakistan can help us to individualize and to develop culturally relevant interventions and strategies for children who struggle with social interactions.

\section{Method}

A correlation research design was conducted to investigate the relationship between theory of mind and peer problems in children. Data were collected by using a non-probability purposive sampling strategy. The sample consisted of children $n=80,42$ from government schools in Islamabad and 38 from the community with age range 4 to 6 years. Theory of mind was assessed through the false belief understanding of the child. In this study, two types of tasks are used in the measurement of false belief. One is a standard location task and the other is unexpected content. Standard false belief tasks are shown to have 0.66 and 0.77 testretest reliability and $0.66 \& 0.88$ internal consistency (Hughes et al., 2000). Hutchins et al. (2012) reported .89 test-retest reliability and 0.98 internal consistencies of standard false beliefs tasks. Construct validity (0.96) of false belief tasks has been established (Wellman \& Liu, 2004). The value of Cronbach Alpha for false belief tasks used in this study was 0.84 .

\section{Materials}

\section{Unexpected Content Task}

Material was used including a box of prince biscuit and a pencil. In the unexpected content task, the experimenter showed a prince biscuit box, and asked the child what was in the box? After answering the question, the participant was shown what was inside the box, and the participant found that it was a pencil inside the box. Then, the experimenter asked the Other Belief Question from the child that what "his friend will think is inside the box after looking at it?" and Self Belief Question that "before opening the box what did you think was inside the box?" Both these questions were asked in counterbalanced order. The last 
question asked was a Reality Question that asked "what is really in the box"? On every correct answer, the child was given 1 and for wrong answer 0 score. In order to pass the Other Belief Question and Self Belief Question, it is important to pass the Reality Question.

\section{Standard Location Task}

Two boxes (green and blue), a horse, a dog, and grass were used in standard location task. In the standard location task, a story was told to the participant with the help of puppets. The experimenter placed two boxes (green and blue) on the table, in front of the child. Then, after introducing the horse with grass, the child was told that "as the horse is tired, it will place his grass in the green box to keep it safe and will go out of the room to sleep". Meanwhile, when the horse was gone, a dog came, took the grass out from the green box, put it in the blue box and went out. Afterwards, the horse woke up and returned to find his grass. The experimenter then asked the child Test Question "where will the horse search his grass?" Then the second question was a memory control question; "where did the horse put his grass first?" Then the reality question was asked "Where is the grass now?" Both the reality and memory control questions were asked alternatively from the participants, in order to counterbalance the order effect. On every correct answer, the child was given 1 and for wrong answer 0 score. In order to pass the Test Question, it is important to pass the Reality and Memory Control Question.

The assessment of peer problems was taken from parents rating on "Strength and Difficulty Questionnaire" (SDQ)-in Urdu version which is a standardized tool and was used to measure peer problems. SDQ-Urdu version has good internal consistency, with Cronbach's alpha of 0.70 and is a reliable and valid measure of behavior and emotions in Pakistani context (Essau et al., 2017).

\section{Aggression subscale of Child Behavior Check List (CBCL)}

The aggression subscale of "Child Behavior Check List" (CBCL) in Urdu version was used to measure aggression. The psychometric properties of CBCL are satisfactory (Achenbach \& Edelbrock, 1991). The internal consistency of CBCL-Urdu version is good with Cronbach alpha of 0.83 (Anjum \& Malik, 2010).

\section{Pilot Study}

The pilot study was conducted initially with sample 10 to check the false belief tasks, so that the examiner could effectively engage the children during the task and to check any difficulty in procedure to assess the theory of mind and any inconvenience in statistical analysis.

\section{Main Study}

SDQ and CBCL questionnaires (both in Urdu versions) were filled by the parents. Tasks to determine theory of mind development were administered individually by the experimenter in school and took approximately 10 minutes per child. Data was also collected from the community setting. The researcher went to the homes of children, where they were administered theory of mind tasks in a separate room which took approximately 10 minutes per child. Parents were asked to fill the SDQ and CBCL questionnaire (both in Urdu versions).

\section{Ethical Consideration}

Ethical considerations as per APA were taken into consideration. Permission from the federal education ministry, Islamabad was taken to collect data from government schools. Information regarding study was given to parents and consent was taken. Ascent from children was also taken. Children who scored higher on SDQ and CBCL, their parents were given references of psychological facilities. Information collected was solely used for research purposes. 


\section{Results}

\section{Table 1}

Descriptive Statistics of Variables of the Study $(N=80)$

\begin{tabular}{lll}
\hline Variables & $\boldsymbol{M}(\boldsymbol{S D})$ & $\boldsymbol{f ( \% )}$ \\
\hline Age & $5.29(0.87)$ & \\
No. of Siblings & $3.35(0.97)$ & \\
Gender & & \\
$\quad$ Female & & $38(47.5 \%)$ \\
$\quad$ Male & & $42(52.5 \%)$ \\
$\quad$ Total & & $80(100.0 \%)$
\end{tabular}

Birth Order

First Child

$15(18.8 \%)$

Middle Child

$52(65.0 \%)$

Last Child

$12(15.0 \%$

Only Child

$1(1.3 \%$

Total

$80(100.0 \%)$

Mother's Education

Less than matric

$26(32.5 \%)$

Matric

$29(36.3 \%)$

Intermediate

$14(17.5 \%)$

BA/BSc

$8(10.0 \%)$

MA/MSc and above

$3(3.8 \%)$

Total

$80(200.0 \%)$

Father's Education

Less than matric

$18(22.5 \%)$

Matric

$33(41.3 \%)$

Intermediate

$13(16.3 \%)$

$\mathrm{BA} / \mathrm{BSc}$

$12(15.0 \%)$

MA/MSc and above

$4(5.0 \%)$

Total

$80(100.0 \%)$

Monthly Income (Rs.)

25275.00

13764.73

Community or School 
School

Community

ToM 1

Fail

Pass

Total

ToM 2

Fail

Pass

Total
$42(52.5 \%)$

$38(47.5 \%)$

$50(62.5 \%)$

$30(37.5 \%)$

$80(100.0 \%))$

ToM 3
Fail
$67(83.8 \%)$
Pass
$13(16.3 \%)$
Total $80(100.0 \%)$

Note. $M=$ Mean, $S D=$ Standard Deviation, $N=$ total no. of participants, ToM= Theory of Mind

Table 1 shows the demographic characteristics of the respondents including age, number of siblings, education level etc. and ToM tasks. Performance on ToM tasks was 0.79 on average, which was low as the maximum score on ToM tasks was 3.

Table 2

Binomial descriptive of Theory of Mind and Age categories $(N=80)$

\begin{tabular}{|c|c|c|c|c|c|c|c|c|c|c|}
\hline \multirow[b]{2}{*}{$\begin{array}{l}\text { Age } \\
\text { (Range) }\end{array}$} & \multirow[b]{2}{*}{ Category } & \multicolumn{3}{|c|}{$\begin{array}{l}\text { Theory of } \\
\text { Mind } 1\end{array}$} & \multicolumn{3}{|c|}{$\begin{array}{l}\text { Theory of } \\
\text { Mind } 2\end{array}$} & \multicolumn{3}{|l|}{$\begin{array}{l}\text { Theory of } \\
\text { Mind } 3\end{array}$} \\
\hline & & $N$ & $\begin{array}{l}\text { Obsvd } \\
\text { prp }\end{array}$ & $p$ & $N$ & $\begin{array}{l}\text { Obsvd } \\
\text { prp }\end{array}$ & $\boldsymbol{P}$ & $N$ & $\begin{array}{l}\text { Obsvd } \\
\text { prp }\end{array}$ & $p$ \\
\hline \multirow[t]{3}{*}{$4.0-4.5$} & Fail & 13 & 0.87 & .007 & 14 & 0.93 & .001 & 15 & 1.00 & 0.00 \\
\hline & Pass & 2 & 0.13 & & 1 & 0.07 & & 0 & & \\
\hline & Total & 15 & 1.00 & & 15 & 1.00 & & 15 & 1.00 & \\
\hline \multirow[t]{3}{*}{$4.6-4.11$} & Fail & 12 & 1.00 & .00 & 12 & 1.00 & .00 & 12 & 1.00 & 0.00 \\
\hline & Pass & 0 & & & 0 & & & 0 & & \\
\hline & Total & 12 & 1.00 & & 12 & 1.00 & & 12 & 1.00 & \\
\hline \multirow[t]{3}{*}{$5.0-5.5$} & Fail & 13 & 1.00 & .00 & 13 & 1.00 & .00 & 13 & 1.00 & 0.00 \\
\hline & Pass & 0 & & & 0 & & & 0 & & \\
\hline & Total & 13 & 1.00 & & 13 & 1.00 & & 13 & 1.00 & \\
\hline \multirow[t]{3}{*}{$5.6-5.11$} & Fail & 7 & 0.54 & 1.00 & 10 & 0.77 & .09 & 10 & 0.77 & 0.09 \\
\hline & Pass & 6 & 0.46 & & 3 & 0.23 & & 3 & 0.23 & \\
\hline & Total & 13 & & & 13 & 1.00 & & 13 & 1.00 & \\
\hline $6.0-6.5$ & Fail & 5 & 0.31 & 0.21 & 11 & 0.69 & .21 & 16 & 1.00 & 0.00 \\
\hline
\end{tabular}




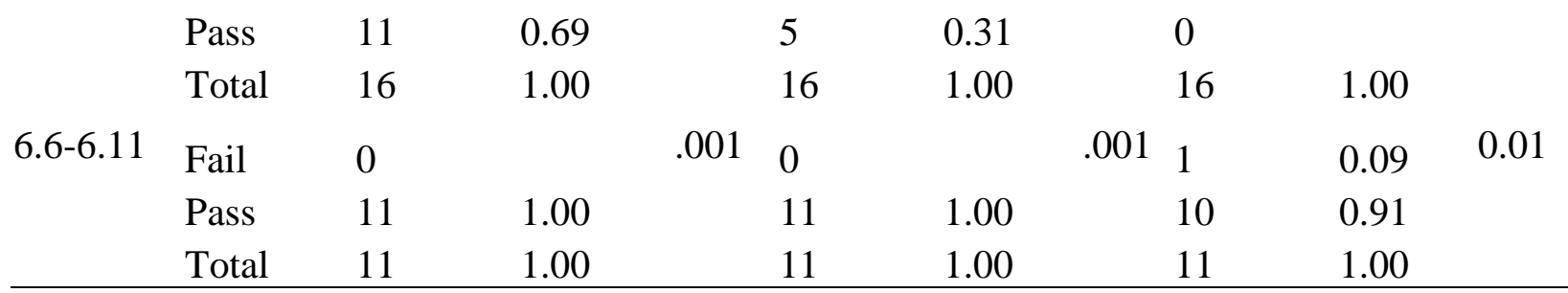

Note. $N=80, p=$ Significance level, obsvd prop= Observed Proportions, ToM $1=$ Standard location task, ToM 2=Deceptive box task (self-belief question), ToM 3=Deceptive box task (other-belief question)

Table 2 represents the results of the Binomial statistics. According to these results, ceiling can be observed in the performance of children of 6.6 years and above age $(p<.01)$, on all the false belief tasks. Children of 6.6 to 6.11 years of age performance were above chance.

Table 3

Contingency Table for Theory of Mind 1(Standard Location), Theory of mind 2 (Deceptive Box task; Self-belief), Theory of mind 3 (Deceptive Box task; Other-belief), and Age categories

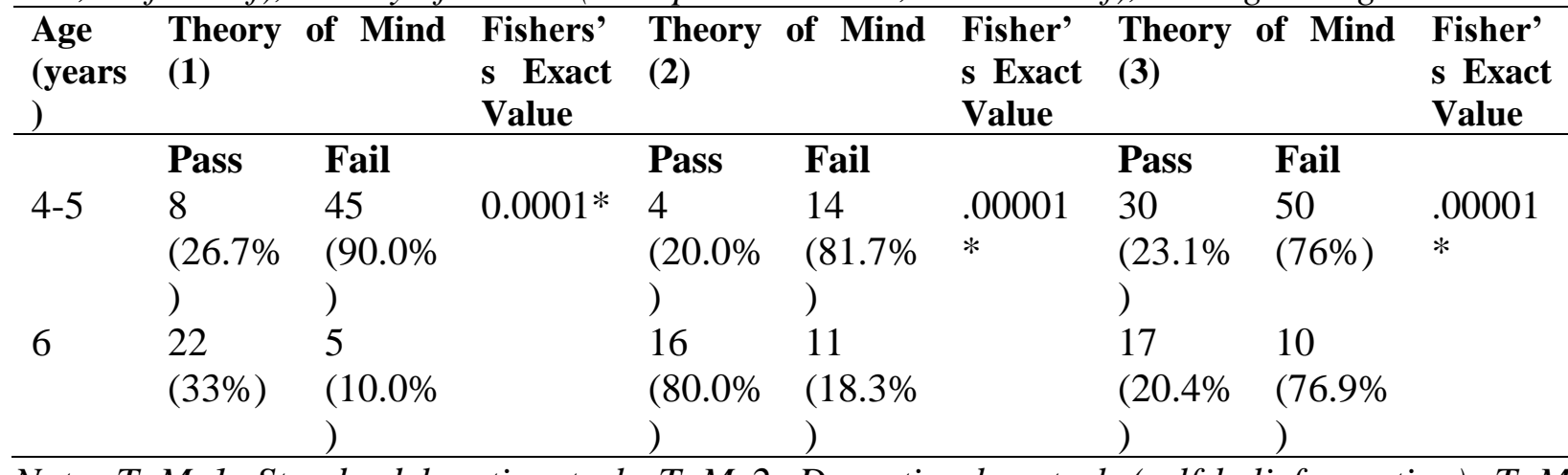

Note. ToM 1=Standard location task, ToM 2=Deceptive box task (self-belief question), ToM $3=$ Deceptive box task (other-belief question)

Table 3 showed that the Fisher exact value for age categories and theory of mind tasks is
.00001 and it is statistically significant, as $p<.05$. 


\section{Table 4}

Pearson Product Moment Correlation between Theory of Mind, Age, No. of Siblings Birth Order, Mother's Education, Father's Education, Monthly Income, Peer Problems, and Aggression $(N=80)$

\begin{tabular}{|c|c|c|c|c|c|c|c|c|c|}
\hline Variables & 1 & 2 & 3 & 4 & 5 & 6 & 7 & 8 & 9 \\
\hline 1. Theory of Mind & - & $.67 * *$ & 0.09 & -0.05 & 0.11 & 0.02 & -0.07 & $-0.28 *$ & -0.07 \\
\hline 2. Age & & - & 0.09 & -0.19 & 0.04 & -0.1 & -0.04 & 0.19 & 0.01 \\
\hline 3. No. of Siblings & & & - & $0.68 * *$ & $-0.28 *$ & $-0.29 * *$ & $-0.29 * *$ & -0.03 & -0.05 \\
\hline 4. Birth Order & & & & - & $-0.25^{*}$ & -0.14 & $-0.22 *$ & $-0.22 *$ & -0.2 \\
\hline 5. Mother's Education & & & & & - & $0.65 * *$ & $0.42 * *$ & 0.05 & -0.01 \\
\hline $\begin{array}{l}\text { 6. Father's } \\
\text { Education }\end{array}$ & & & & & & - & $0.43 * *$ & 0.03 & -0.06 \\
\hline 7. Monthly Income & & & & & & & - & 0.08 & 0.13 \\
\hline 8. Peer Problems & & & & & & & & - & 0.12 \\
\hline 9.Aggression & & & & & & & & & - \\
\hline
\end{tabular}

Note. $N=80 .{ }^{*} p<0.05,{ }^{*} * p<0.01$. (Gender correlated with ToM $r=.31$ )

Table 4 depicts Pearson product correlation analysis showed that there was a positive significant relationship between performance on theory of mind tasks and age, $r=.67$ ( $p<.001)$, number of siblings and birth order, $\mathrm{r}=.68 \quad(p<.001)$, number of siblings and mother's education as $\mathrm{r}=-.28(p<.05)$, number of siblings and father's education $(\mathrm{r}=-.29$, $p<.001)$, and number of siblings and monthly income $(\mathrm{r}=-.29(p<.001)$, birth order and peer problems $\mathrm{r}=-.22 \quad(p=.05)$. There was no significant correlation between aggression and peer problems $(\mathrm{r}=0.12, p>.05)$. Gender and theory of mind ability is also positively correlated, $(\mathrm{r}=.31(p<.001)$.

Table 5

Predictors of Peer Problems in Children $(N=80)$

\begin{tabular}{|c|c|c|c|c|}
\hline \multirow[b]{3}{*}{ Variables } & \multicolumn{4}{|c|}{ Peer Problems } \\
\hline & & Mod & & \\
\hline & B & B & S.E & 95\% C.I \\
\hline Constant & $4.48 * * *$ & & .52 & $3.44-5.51$ \\
\hline ToM Continuous & -.45 & -.29 & .16 & $-.79--.13$ \\
\hline Birth Order Continuous & -.45 & -.23 & .20 & $-.86--.05$ \\
\hline $\mathrm{R}^{2}$ & 13 & & & \\
\hline$\Delta \mathrm{R}^{2}$ & .13 & & & \\
\hline
\end{tabular}


Note. $*=p<.05, * *=p<0.1, * * *=p<.001, \mathrm{CI}=$ confidence interval, $\mathrm{B}=$ Unstandardized Regression Coefficient, $\beta=$ Standard Regression Coefficient, $\Delta \mathrm{R}^{2}=$ Change in $\mathrm{R}^{2}, \mathrm{CI}=$ Confidence Interval, ToM=Theory of mind

Table 5 depicts multiple regression. Results showed that theory of mind significantly

\section{Discussion}

This study builds on intermediate approach of ToM development, according to which universalism and relativism are two ends of the continuum (Alivah-Naveh, 2019). This study is one step contribution to our understanding of age at which ToM develops in Pakistani children which is 6 years and 6 months, during which the performance on false belief tasks is above chance. This is in line with the meta-analysis conducted by Wellman in 2014, according to which all normally developing children eventually develop false belief understanding, with the difference that some develop it late and some develop it early. The trajectory of below chance incorrect judgment to above chance correct judgment is followed universally, but the age range is different in different cultures, some develop it at four, and some at six to seven years of age (Wellman, 2014) or even after 7 (De Gracia et al., 2016; Mayer \& Taruble, 2012), supporting the universality of ToM with different age range in different cultures (relativism).

One of the reasons for such a finding could be that children in Pakistan live below the poverty line and there is a difference in ToM development in children from affluent communities and poor ones, as a study in India confirmed (Baidee, 2013). Difference in development of theory of mind can be attributed to culture and language environment as well. Variations caused by culture or social factors are difficult to underpin, as to understand exactly what cultural variables affect theory of mind is hard. There is a growing body of research indicating that family interactions and predicted the variation in the peer problems, $F(1,78)=6.84, p<.000, \mathrm{R}^{2}=13 \%$.

environment, which are shaped by culture, impact social understanding of children (Kuntoro et al., 2017). One of the social factors related to theory of mind is parental education, both mother's higher education (Ensor \& Hughes, 2008; Hughes, 2011) and father's higher education (Shahaeian, 2015) are positively associated with children's ToM development. Mother's low education is related to child's low IQ, which can indirectly impact ToM development, and higher maternal education is associated with children's cognitive and language ability, which is associated with greater ToM understanding (Pears \& Moses, 2003). According to the results of this study, mother's and father's education was not associated with theory of mind. The reason could be that most of the mothers and fathers had 10 years of education, which is not higher education. In future, this factor could be investigated in depth by involving parents with higher education. Another explanation for developmental lag can be explained through language development. Language development also plays an important role in social understanding (Villiers et al., 2014), as it is through language that enables a child to engage in culture, to interact in social situations and participate in conversations (Nelson, 2005). According to literature, it is these activities that influence ToM development and enhance false belief understanding (Milligan et al., 2007). Choosing different verbs in false belief tasks can impact performance level of children (Lee et al., 1999). According to Nawaz et al. (2014), children in Pakistan who use cognitive terms during conversations are 
better at understanding others' minds. By constructing mental state terms, they understand the mind of others, which also supports the idea of general language ability of children (Nawaz et al., 2014), as language mastery, instead of conversational input, helps children to develop better ToM abilities (de Villiers \& de Villiers, 2014). Understanding of complex syntactic structure is important for representing the understanding of false belief, like knowledge of sentential complementation (de Villiers \& Pyers, 2002). The example of sentential complement is; Sally thinks her chocolate is in the cupboard. This is a crucial sentence where the main clause reported is true but the underlying proposition is false. Hence, these syntactic structures are a format in language through which children represent the understanding of false belief (de Villiers, 2005). However, this study could not take into account general language ability of children, due to non-availability of culturally specific, standardized measures of language ability. Further exploration of language development is needed to reach any definite conclusion.

The next hypothesis of the study was about correlation between ToM and peer problems. It was hypothesized that there will be a negative relationship between theory of mind and peer problems. According to the results of this study, there is a significant negative relationship between theory of mind ability and peer problems which means that when theory of mind ability increases, peer problems decrease, and impairment in theory of mind is associated with an increase in peer relationship problems. These results are in line with the research conducted by Mizokawa and Koyasu (2013). According to the results, children with advanced performance on false belief tasks had fewer peer problems. The results were similar even when the language ability was controlled. In this study, peer problems were rated by parents, due to the lack of a teacher version of Strength and Difficulty Questionnaire. Peer problems were rated by parents in other studies as well (Schlotz et al., 2010).

As per the results of the present study, peer problems and performance on false belief tasks are significantly negatively correlated. This is due to the reason that children who face difficulty at false belief tasks, is because they understand other's mental state better, which can be related to better communication abilities, hence, they get along with peers better, and face lesser peer problems (Mizokawa \& Koyasu, 2013). Some of the factors like cooperation, better communication quality, prosocial behavior and effective group participation, which are necessary for better relationships may at least be partially linked with ToM (Begeer et al., 2011) due to which children with better ToM ability, might have better peer relations, whereas children with reduced mental state understanding are regarded as less capable socially, and not liked by their peers (Slaughter et al., 2015).

These findings of the study, point to the fact that false belief comprehension is the key for better adaptation to social world (Hughes, 2011), and social competence necessary while interacting with peers is linked with the understanding of other's mind based on theory of mind ability (Peterson et al., 2016). Although ToM understanding predicts peer problems significantly as per results, the overall effect size is small, as TOM contributes to only $7 \%$ variance in peer problems. One of the reasons could be the sample size of the study and including larger sample size might have different results (Brooks et al., 2011). Another plausible reason could be that having a better understanding of other's mind or mental states, does not confirm the use of ToM abilities in social interactions (Hughes, 2011). There is a variable that mediates the relationship between ToM and peer 
problems, which is prosocial behavior (Slaughter et al., 2015), as prosocial behavior is an important consequence of theory of mind, because better peer relations are linked with theory of mind that is used in socially helpful and cooperative behaviors (Caputi et al., 2012). The use of prosocial behavior stemming from ToM development with peers, may be crucial for saving the child from facing peer problems (Caputi et al., 2012). Therefore, future studies can incorporate mediating variables like prosocial behavior, and investigate its link with ToM and peer relations.

Knowing special populations like children with autism have impaired false belief understanding, implicating their social lives (Rajendran \& Mitchell, 2007), so it is important to see if the delay in ToM understanding is due to cultural difference, implicated by the child's language environment or other social factors. Nevertheless, educationists need to focus on this if this is a developmental delay. Furthermore, these findings ascertain that social development in Pakistan is behind and could be improved through ToM training. However, further research is needed to know if this delay affects later social and cognitive milestones of children.

\section{Conflict of Interest}

There is no conflict of interest declared by authors.

\section{Source of Funding}

The authors declared no source of funding.

\section{References}

Achenbach, T. M., \& Edelbrock, F. C. (1991). Manual for the child behavior checklist and revised child behavior profile. Burlington, VT: University of Vermont, Department of Psychiatry.

Alival-Naveh, E., Rothschild-Yakar, L., \& Kurman, J. (2019). Keeping culture in mind: A systematic review and initial conceptualization of mentalizing from a cross-cultural perspective. Clinical Psychology: Science and Practice, 26(4), e12300. https://doi.org/10.1111/cpsp.12300

Anjum, N., \& Malik, F. (2010). Parenting practices in mothers of children with ADHD: Role of stress and behavioral problems in children. Pakistan Journal of Social and Clinical Psychology, 8(1), 18-38. http://www.gcu.edu.pk/Soc\&ClinPsy Jour.htm

Astington, J. W., Margaret, J., \& Edward, M. A. (2010). The development of theory of mind in early childhood. Encyclopedia on early childhood development. 1-4. http://www.childencyclopedia.com/socialcognition/accordingexperts/develop ment-theory-mind-early-childhood

Baidee, S. (2013). World development indicators (p. 29). Washington, DC: International Bank for Reconstruction and Development.

Brooks, S. P., Gelman, A., Jones, G. L., \& Meng, X.-L. (Eds.). (2011). Handbook of Markov Chain Monte Carlo. Boca Raton, FL: Chapman \& Hall/CRC Press.

Callaghan, T., Rochat, P., Lillard, A., Claux, M. L., Odden, H., Itakura, S., \& Singh, S. (2005). Synchrony in the onset of mental-state reasoning: Evidence from five cultures. Psychological Science, 16, 378-384.

Campbell, S. B., \& von Stauffenberg, C. (2008). Child characteristics and family processes that predict behavioral readiness for school. In A Crouter \& A. Booth (Eds.) Early disparities in school readiness: How do families contribute to successful and unsuccessful transitions to 
school. Mahwah, N.J.: Lawrence Erlbaum.

Caputi, M., Lecce, S., Pagnin, A., \& Banerjee, R. (2012). Longitudinal effects of theory of mind on later peer relations: The role of prosocial behavior. Developmental Psychology, 48 (1), 257-270. DOI: 10.1037/a0025402

Begeer, S., Gevers, C., Clifford, P., Verhoeve, M., Kat, K., Hoddenbach, E., \& Boer, F. (2011). Theory of Mind training in children with autism: a randomized controlled trial. Journal of autism and developmental disorders, 41(8), 9971006.

https://doi.org/10.1007/s10803-0101121-9

Chen, X., Huang, X., Wang, L., \& Chang, L. (2012). Aggression, peer relationships, and depression in Chinese children: a multi wave longitudinal study. Journal of Child Psychology and Psychiatry 53, 1233 1241.DOI:10.1111/j.14697610.2012. 02576.x

De Gracia, M. R., de Rosnay, M., \& Peterson, C. C. (2016). A cultural conundrum: Delayed false belief understanding in Filipino children. Journal of CrossCultural Psychology, 47, 929-940.

De Villiers, J. G. (2005). Can language acquisition give children a point of view? In J. W. Astington \& J. Baird (Eds.), Why Language Matters for Theory of Mind (pp. 186- 219).

New York, NY: Oxford University Press.

De Villiers, J. G., \& De Villiers, A. P. (2014). The role of language in development oftheory of mind. Top Language Disorders. 34, 4. 313-328.

de Villiers, J. G., Hollebrandse, B., \& Hobbs, K. (2014). Recursive complements and propositional attitudes. In $\mathrm{T}$.
Roeper, \& P. Speas (Eds.), Recursion. Berlin:

Springer Verlag.

De Villiers, J. G., \& Pyers, J. E. (2002). Complements to cognition: A longitudinal study of the relationship between complex syntax and false-belief-understanding. Cognitive Development, 17, 10371060.

Devine, R. T., White, N., Ensor, R., \& Hughes, C. H. (2016). Theory of mind in middle childhood:

Longitudinal associations with executive function and social competence. Developmental Psychology, $52 \quad 758-771$. DOI: $10.1037 / \mathrm{dev} 0000105$

Ensor, R., \& Hughes, C. (2008). Content or connectedness? Mother child talk and early social understanding. Child Development, 79, 201-216. DOI:10.1111/j.14678624.2007.0112 $0 . \mathrm{x}$

Hughes, C. (2011). Social understanding and social lives: From toddlerhood through to the transition to school. New York: Psychology Press.

Hughes, C., Adlam, A., Happe, F., Jackson, J., Taylor, A.,\& Caspi, A. (2000). Good test retest reliability for standard and advanced false-belief tasks across a wide range of abilities. Journal of Child Psychology \& Psychiatry, 41, 483-490.

Hutchins, T. L., Prelock, P. A., \& Bonazinga, L. (2012). Psychometric evaluation of the theory of mind inventory (ToMI): A study of typically developing children and children with autism spectrum disorder. Journal of Autism Developmental Disorder, 42, 327-41.

Essau, C. A., Qadir, F., \& Maqsood, A. (2017). Factor structure of the Urdu version of the strengths and 
difficulties questionnaire in Pakistani adolescents. Act of Psychopathy, 3-1. DOI: 10.4172/2469-6676.100076

Kuntoro, I. A., Peterson, C. C. \& Slaughter, V. (2017). Culture, parenting and children's theory of mind development in Indonesia. Journal of Cross-Cultural Psychology,48(9), $1389-1409$.

Lee, K., Olson, D. R., \& Torrance, N. (1999). Chinese children's under-standing of false beliefs: The role of language. Journal of Child Language, 26, 1-21.

Liu, D., Wellman, H. M., Tardif, T., \& Sabbagh, M. A. (2008). Theory of mind development in Chinese children: a meta-analysis of falsebelief understanding across cultures and languages. Developmental psychology, 44(2), 523-531. https://doi.org/10.1037/00121649.44.2.523

Malle, B. (2018). Theory of mind. In R. Biswas-Diener \& E. Diener (Eds), Noba textbook series: Psychology Champaign, IL: DEF publishers. DOI:nobaproject.com

Mayer, A., \& Trauble, B. (2012). Synchrony in the onset of mental state understanding across cultures? International Journal of Behavioral Development, 37, 21-28.

Milligan, K., Astington, J. W. \& Dack, L. A. (2007). Language and theory of mind: Meta-analysis of the relation between language ability and falsebelief understanding. Child Development, 78, 622-646.

Mizokawa, A., \& Koyasu, M. (2013). Young children's moral judgments about pretend crying: Associations with mental-state understanding. Psychologia: an international journal of psychology in the Orient 56(4):223-236.

DOI: $10.2117 /$ psysoc.2013.223
Nawaz, S., Hanif, R., \& Lewis, C. (2014). Theory of mind' development of Pakistani children: Do preschoolers acquire an understanding of desire, pretence and belief in a universal sequence? European Journal of Developmental

Psychology, 12(2):177-188. DOI: 10.1080/17405629.2014.973843

Nawaz, S., Hanif, R., \& Lewis, C. (2015). 'Theory of mind' development of Pakistani children: Do preschoolers acquire an understanding of desire, pretense and belief in a universal sequence? European Journal of Developmental Psychology, 12(2), 177-188. DOI: 10.1080/17405629.2014.973843

Nawaz, S., \& Lewis, C. (2017). Mother-child conversation and social understanding in Pakistan. International Journal of behavioral development. 10.DOI:10.1177/0165025417741365

Nelson, K. (2005). Language pathways into the community of minds. In: Astington J. W, Baird J, editors. Why Language Matters for Theory of Mind. Oxford University Press; New York: 26-49.

Oh, S., \& Lewis, C. (2008). Korean preschoolers' advanced inhibitory control and its relation to other executive skills and mental state understanding. Child Development, 79(1), 80-99. DOI: $10.1111 / \mathrm{j} .1467-$ 8624.2007.01112.x

Patnaik, B. (2008). Children's theory of mind: educational, school and instructional implications. Journal of the Indian Academy of Applied Psychology, 34(2), 329- 336.

Pears, K. C., \& Moses, L. J. (2003). Demographics, parenting, and theory of mind in preschool children. 
Social Development, 12, 1-20. DOI:10.1111/1467-9507.00219

Peterson, C., Slaughter, V., Moore, C., \& Wellman, H. M. (2016). Peer social skills and theory of mind in children with autism, deafness, or typical development. Developmental Psychology, 52(1), 46-57. DOI: $10.1037 / \mathrm{a} 0039833$

Rajendran, G., \& Mitchell, P. (2007) Cognitive theories of autism. Developmental Review, 27, 224-260.

Schlotz, W., Jones, A., Phillips, D. I. W., Gale, C. R., Robinson, S. M., \& Godfrey, K.M. (2010). Lower maternal folate status in early pregnancy is associated with childhood hyperactivity and peer problems in offspring. Journal of Child Psychology and Psychiatry. 51(5), 594- 602. DOI:10.1111/j.1469 7610.2009.02182.x

Shahaeian, A. (2015). Sibling, family and social influences on children's theory of mind understanding. Journal of Cross-Cultural
Psychology, $46 \quad$ (6), 805-820. DOI:10.1177\%2F002202211558389 7

Slaughter, V., Imuta, K., Peterson, C. C., \& Henry, J. D. (2015). Meta-analysis of theory of mind and peer popularity in the preschool and early school years. Child Development, 86(4), 11591174.

Wellman, H. M. (2007). Understanding the psychological world: Developing a theory of mind Blackwell Handbook of Childhood Cognitive Development (pp. 167-187): Blackwell Publishers Ltd.

Wellman, H. M. (2012). Making minds. Oxford, UK: Oxford University Press.

Wellman, H. M. (2014). Making minds: How theory of mind develops. Oxford University Press.

Wellman, H. M., \& Liu, D. (2004). Scaling of theory-of-mind tasks. Child Development,75, 523DOI:10.1111/j.14678624.2004.00691.x 